\title{
Rapid expansion of the descending aorta following total arch replacement with a FROZENIX-open stent-graft for thoracic aortic aneurysm in a patient with Marfan syndrome: a case report
}

\author{
Tatsuya Nakao, Shoichi Tsuda^, Nao Kume, Yuki Ikegaya, Yosuke Kitanaka \\ Department of Cardiovascular Surgery, New Tokyo Hospital, Matsudo, Chiba, Japan \\ Correspondence to: Tatsuya Nakao. Department of Cardiovascular Surgery, New Tokyo Hospital, 1271 Wanagaya, Matsudo, Chiba, 270-2232, Japan. \\ Email: tatsuyacvs1228@gmail.com.
}

\begin{abstract}
Frozen elephant technique has been used to treat extensive aortic pathology (EAP) in either one or two stage procedures. Rapid expansion of the downstream aorta following frozen elephant technique was not reported. A 28-year-old man was referred to our clinic with a dilated abdominal aorta. He had a family history of Marfan syndrome. Contrast-enhanced computed tomography of the body revealed an EAP, including annuloaortic ectasia, an aortic aneurysm in the distal thoracic aorta, and a thoracoabdominal aortic aneurysm (Crawford type IV). Transthoracic echocardiography demonstrated mild aortic regurgitation. The patient underwent aortic root replacement (Bentall procedure) and total arch replacement (TAR) with the frozen elephant technique using a FROZENIX-open stent-graft. On postoperative day 8, the patient reported backache; computed tomography of the chest revealed the expansion of the descending aorta and pericardial effusion. We suspected an impending rupture of the thoracic aortic aneurysm and cardiac tamponade. He underwent an emergent reoperation, including a pericardial window for drainage and thoracic endovascular aortic repair (TEVAR) for the rapid expansion of the thoracic descending aorta. The postoperative course was uneventful. To the best of our knowledge, this is the first case of rapid expansion of the descending aortic aneurysm following TAR with the frozen elephant technique, which was successfully treated with an additional endovascular re-intervention. Endovascular re-intervention could be safely completed because of the stented graft used for the frozen elephant technique. Although frozen elephant technique (FET) is technically favorable for additional TEVAR, close follow-up of all patients undergoing FET is warranted.
\end{abstract}

Keywords: Extensive aortic pathology (EAP); frozen elephant technique; re-intervention; case report

Received: 12 April 2020; Accepted: 17 September 2020; Published: 20 July 2022.

doi: 10.21037/jovs-20-102

View this article at: http://dx.doi.org/10.21037/jovs-20-102

\section{Introduction}

Extensive aortic pathology (EAP) can be managed successfully since the introduction of the two-stage elephant trunk principle in 1983 by Borst (1). This involves inserting the extension of the prosthetic graft segment in the distal arch in the first stage procedure, which is the total arch replacement (TAR) and using the extended graft for the proximal anastomosis in the second stage procedure for the descending aorta. Interval rupture (IR), the rupture before the second surgery for the remaining aortic pathology, has been an issue, possibly due to the free-floating nonstented prosthetic "elephant trunk" segment. The frozen elephant technique (FET), a technique involving the use of a stented open graft as the elephant trunk segment, has been introduced to prevent IR and make the second procedure feasible if needed (2). The rapid expansion of

^ ORCID: 0000-0001-6912-4472. 


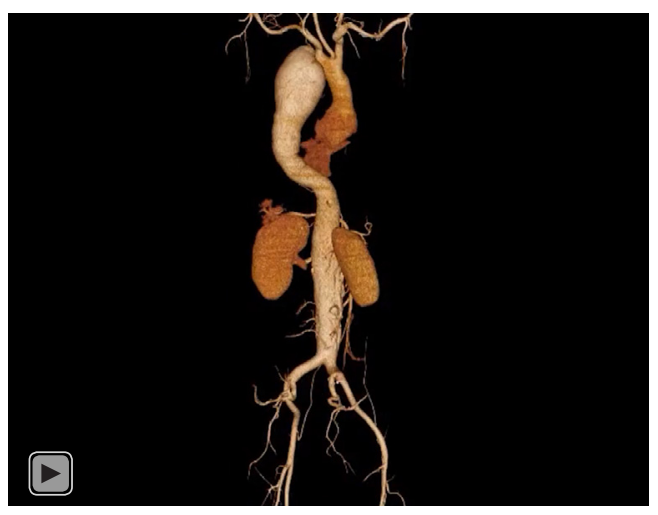

Video 1 Preoperative computed tomographic angiography demonstrated extensive aortic pathology.

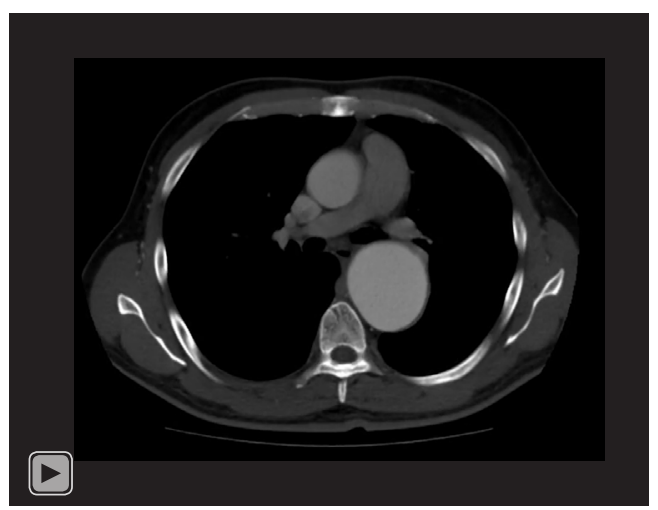

Video 2 Contrast-enhanced computed tomography revealed an extensive aortic pathology including annuloaortic ectasia measuring $54 \mathrm{~mm}$, a dilated aorta of the distal aortic arch, measuring $61 \mathrm{~mm}$, and a dilated thoracoabdominal aorta (Crawford type IV), measuring $45 \mathrm{~mm}$.

the descending aorta warranting a second intervention to prevent rupture has been reported following TAR with the conventional elephant trunk (CET) (3), but not FET. We herein report a case of rapid expansion of the descending aorta following TAR with FET, which was successfully treated by reoperation with an additional thoracic endovascular aortic repair (TEVAR). We present the following case in accordance with the CARE reporting checklist (available at https://jovs.amegroups.com/article/ view/10.21037/jovs-20-102/rc).

\section{Case presentation}

A 28-year-old man was referred to our clinic owing to a dilated aorta. He had a family history of Marfan syndrome and aortic rupture. He denied any symptoms indicating acute aortic syndrome. Contrast-enhanced computed tomography of the body revealed an EAP including annuloaortic ectasia measuring $54 \mathrm{~mm}$ in diameter, a thoracic aortic aneurysm of the distal arch with aortic dissection, measuring $61 \mathrm{~mm}$, and a thoracoabdominal aortic aneurysm (Crawford type IV, measuring $45 \mathrm{~mm}$ ) (Videos 1,2). Transthoracic echocardiography revealed normal systolic function and mild aortic regurgitation. We planned a multi-stage repair of the EAP.

He underwent Bentall procedure and TAR with FET as the first stage procedure. Intraoperative transesophageal echocardiography demonstrated mild aortic regurgitation and good systolic function. Following median sternotomy, cardiopulmonary bypass (CPB) was instituted with an $8 \mathrm{~mm}$ FUSION vascular graft (Maquet Cardiovascular, Wayne, NJ, USA) anastomosed to the left subclavian artery (LSCA) and a distal ascending aortic cannula and a two-stage right atrial cannula. A cannula for retrograde cardioplegia (CP) was placed in the coronary sinus and the vent in the left ventricle via the left superior pulmonary vein. The patient's body temperature was lowered to $30^{\circ} \mathrm{C}$. Following an aortic cross-clamp, an aortotomy was performed for inspection. Antegrade CP was administered selectively via the coronary artery ostia. Aortic root replacement was performed using a composite graft consisting of a mechanical bileaflet prosthesis On-X Prosthetic Heart Valve $25 \mathrm{~mm}$ (CryoLife, Inc. Kennesaw, GA, USA) and $30 \mathrm{~mm}$-Gelweave Valsalva Grafts (TERUMO, Ann Arbor, MI, USA). After completing the composite graft replacement with the aortic root, the coronary buttons were anastomosed with the graft. The patient's body temperature was further lowered to $25{ }^{\circ} \mathrm{C}$, and hypothermic circulatory arrest was established. Selective antegrade cerebral perfusion was added. The dilated aorta was resected between the brachiocephalic artery (BCA) and the left carotid artery (LCA), with the root of the LSCA clamped. Selective antegrade cerebral perfusion was instituted via cannulas in the BCA, LCA, and the graft anastomosed to the LSCA with the root of the LCA severed and closed. We used the Frozenix J Graft (Japan Lifeline, Tokyo, Japan), $27 \mathrm{~mm} \times 12 \mathrm{~cm}$, an open stent-graft, composed of a proximal vascular polyester prosthesis and distal stented part made of nitinol wire. After the curvature of the tip was adjusted, the open stentgraft was gently introduced into the distal descending aorta through the narrowed distal aortic arch, measuring $21 \mathrm{~mm}$. Once the open stent-graft was deployed at the 


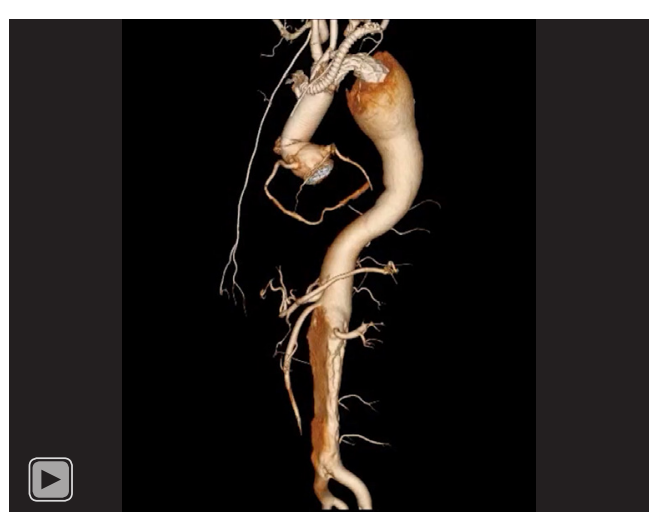

Video 3 3D-reconstruction taken on POD 8 demonstrated rapid expansion of the descending aorta. POD, postoperative day.

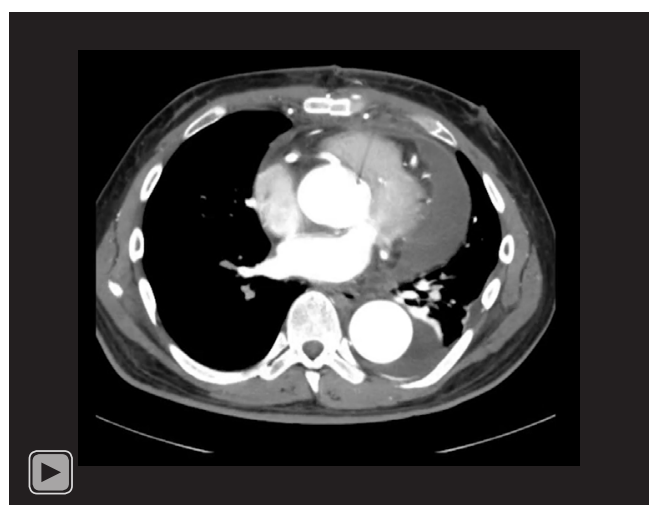

Video 4 Axial CT taken on POD 8 revealed pericardial effusion as well. POD, postoperative day.

appropriate position and confirmed by transesophageal echocardiography, the proximal vascular part was cut off. The distal anastomosis was directly performed between the descending aorta, the Frozenix, and the four-branched vascular graft Triplex (Terumo Co., Tokyo, Japan). The anastomosis was first fixed with buttressed sutures circumferentially and completed by multiple running sutures. After distal perfusion was re-instituted through the side branch of the graft anastomosed to the distal aorta with the proximal end of the graft clamped, the branches of the graft were anastomosed with the BCA and LCA. Rewarming was then initiated. The graft anastomosed to the LSCA was introduced via the thoracic cavity and anastomosed to the third branch of the four-branched graft. Then, the graft was proximally anastomosed with the ascending aorta. After securing hemostasis, de-airing was performed through a cannula placed in the aortic root. By removing the retrograde $\mathrm{CP}$ cannula, the aortic cross-clamp was removed. After de-airing and securing hemostasis, the $\mathrm{CPB}$ was weaned. Transesophageal echocardiography demonstrated the completion of de-airing and good cardiac function. The cannulas were removed, and the side branch used for the inflow of the CPB was ligated. The chest was closed. The patient was shifted to the intensive care unit from the operative room with a favorable hemodynamic status.

The patient was recovering well until he reported backache on the postoperative day 8 . Transthoracic echocardiography revealed massive pericardial effusion with a sign of cardiac tamponade. Chest computed tomography revealed rapid expansion of the descending aorta (Videos 3,4). Based on the clinical symptoms and imaging findings, we diagnosed an impending rupture of the descending thoracic aorta and pericardial tamponade. An emergency reoperation was performed, including a pericardial window for drainage and an additional TEVAR. Under general anesthesia, a mini-thoracotomy at the fourth intercostal space was made, which revealed tense thickened pericardium underneath the thoracotomy. The pericardial window was made, and serosanguinous pericardial effusion was drained. No active bleeding was noted in the pericardial cavity. The artery was accessed via the right common femoral artery. Intraoperative aortography demonstrated turbulent and accelerated flow through the narrowed area in the aortic arch (Video 5). An additional TEVAR was performed using two pieces of Valiant thoracic endograft, Proximal FreeFlo Straight, 30-30-200 and 32-32-150 (Medtronic, Santa Rosa, CA, USA) deployed in zone 3. Postprocedural aortography revealed no endoleak (Video 6). The patient was shifted from the operation theatre in a good hemodynamic state. The postoperative course was uneventful. Postoperative computed tomography demonstrated no endoleak or further dilatation of the descending aorta (Videos 7,8).

All procedures performed in this study were in accordance with the ethical standards of the institutional and/or national research committee(s) and with the Helsinki Declaration (as revised in 2013). Written informed consent was obtained from the patient for publication of this case report and accompanying images. A copy of the written consent is available for review by the editorial office of this journal.

\section{Discussion}

Although the elephant trunk technique enables surgeons 


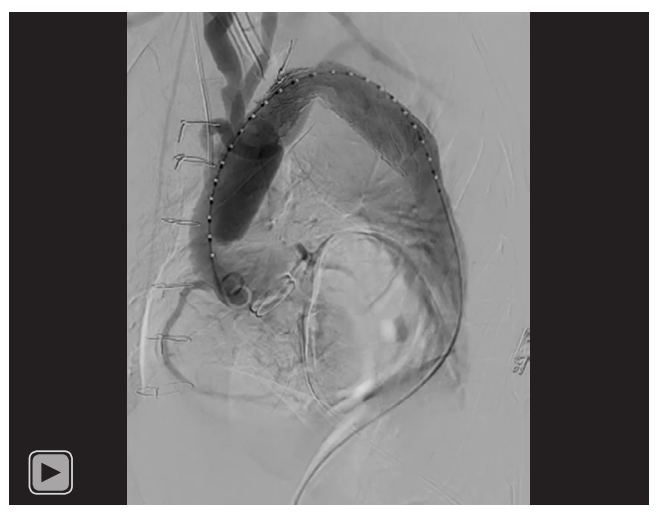

Video 5 Intraoperative aortography at the second operation demonstrated turbulent and accelerated flow through the narrowed area on the aortic arch.

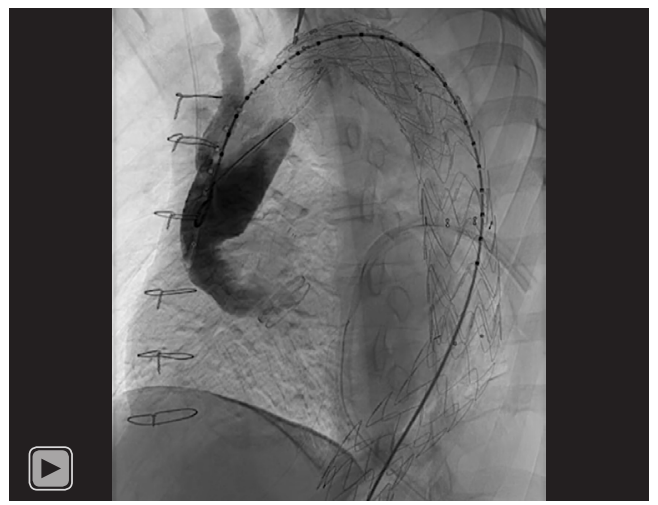

Video 6 Postprocedural aortography demonstrated no endoleak after the completion of the emergent thoracic endovascular aortic repair.

to handle an EAP in two stages, IR, the rupture of the remaining aortic aneurysm awaiting the second procedure, is among the major complications. Rapid expansion after the first procedure poses a risk of rupture, which warrants urgent intervention. Rapid expansion of the descending aorta has been reported after TAR with CET (3), but not after TAR with FET. To the best of our knowledge, this is the first case report of rapid expansion after TAR with FET, which was successfully treated by an additional TEVAR.

The possible mechanisms of rapid expansion include a poor sealing capacity of the non-stented graft of the elephant trunk, laceration of the aorta by the free-floating of the graft, increased wall stress in the systolic phase due to the retrograde flow into the peri-graft lumen (4),

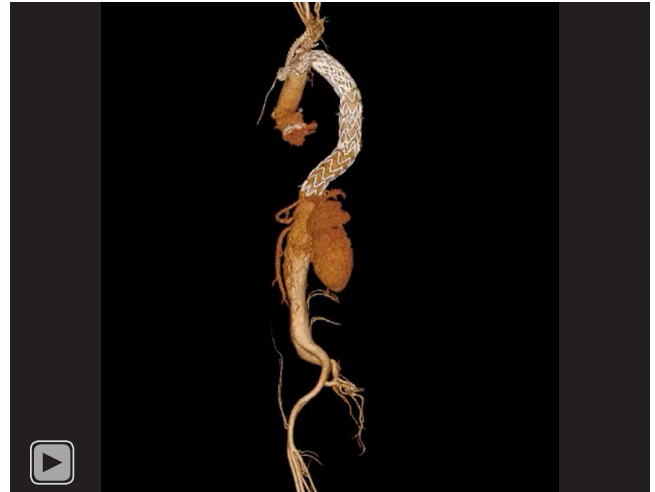

Video 7 3D-reconstruction after an additional thoracic endovascular aortic repair.

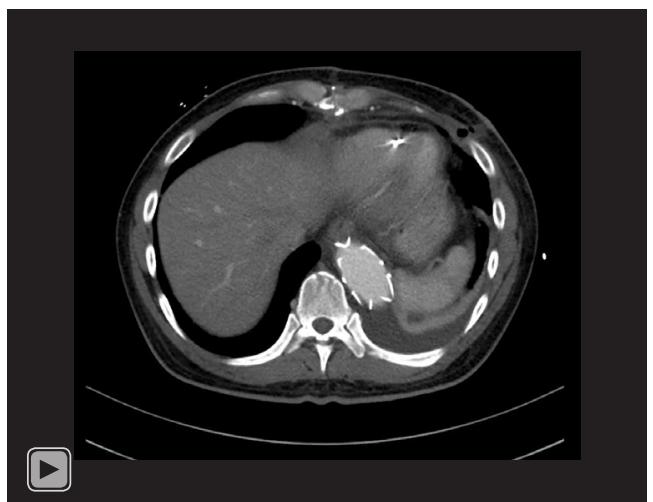

Video 8 Axial CT after the thoracic endovascular aortic repair demonstrated no endoleak.

accelerated flow through the distorted elephant trunk (5), and aneurysm-induced disseminated intravascular coagulation (6). In this patient, the possible mechanisms of rapid expansion include a fragile aortic wall due to Marfan syndrome and an accelerated flow through the stented graft. We used a $27 \mathrm{~mm}$ stented open graft for the distal aortic arch with a narrow dissected distal stump measuring $21 \mathrm{~mm}$. We secured the anastomosis and avoided ballooning of the open stent at the distal end. However, aortography at the reoperation revealed accelerated flow through the narrowed aortic arch mimicking coarctation of the aorta (Video 5), which might have affected the diseased wall of the descending aorta.

The rapid expansion of the descending aorta was successfully treated by an additional TEVAR. Although 
FET may cause positive remodeling in the downstream aortic pathology potentially leading to the whole aortic pathology being treated in one stage, aortic re-interventions after FET are common (7). However, re-interventions after FET are associated with acceptable morbidity and mortality compared with those after CET. TEVAR following TAR with CET is technically challenging because of the difficult passage of the guidewire due to possible flapping and kinking and difficult decisions regarding the landing zone to deploy for TEVAR. Moreover, CET has a high risk of generating a type III endoleak between CET and TEVAR graft. FET allows smooth passage of the GW, with an excellent proximal landing zone at secondary stent-graft deployment. As a result, the outcomes after secondary TEVAR following TAR with FET are excellent as compared to those after TAR with CET.

\section{Conclusions}

This is the first case report of rapid expansion of the descending aorta following TAR with FET, which was successfully treated with immediate reoperation with an additional TEVAR. Although FET is technically favorable for additional TEVAR, aortic re-interventions are common. Close follow-up of all patients undergoing FET is warranted.

\section{Acknowledgments}

Funding: None.

\section{Footnote}

Provenance and Peer Review: This article was commissioned by the Guest Editors (Roberto Di Bartolomeo, Davide Pacini, Mohamad Bashir) for the series "Best Video Presentation Prize for the 10th Postgraduate Course on 'Surgery of the Thoracic Aorta' in Bologna" published in Fournal of Visualized Surgery. The article has undergone external peer review.

Reporting Checklist: The authors have completed the CARE reporting checklist. Available at https://jovs.amegroups. com/article/view/10.21037/jovs-20-102/rc

Conflicts of Interest: All authors have completed the ICMJE uniform disclosure form (available at https://jovs. amegroups.com/article/view/10.21037/jovs-20-102/coif).
The series "Best Video Presentation Prize for the 10th Postgraduate Course on 'Surgery of the Thoracic Aorta' in Bologna" was commissioned by the editorial office without any funding or sponsorship. TN is a proctor of Frozenix, JAPAN Lifeline. The authors have no other conflicts of interest to declare.

Ethical Statement: The authors are accountable for all aspects of the work in ensuring that questions related to the accuracy or integrity of any part of the work are appropriately investigated and resolved. All procedures performed in this study were in accordance with the ethical standards of the institutional and/or national research committee(s) and with the Helsinki Declaration (as revised in 2013). Written informed consent was obtained from the patient for publication of this case report and accompanying images. A copy of the written consent is available for review by the editorial office of this journal.

Open Access Statement: This is an Open Access article distributed in accordance with the Creative Commons Attribution-NonCommercial-NoDerivs 4.0 International License (CC BY-NC-ND 4.0), which permits the noncommercial replication and distribution of the article with the strict proviso that no changes or edits are made and the original work is properly cited (including links to both the formal publication through the relevant DOI and the license). See: https://creativecommons.org/ licenses/by-nc-nd/4.0/.

\section{References}

1. Borst HG, Walterbusch G, Schaps D. Extensive aortic replacement using "elephant trunk" prosthesis. Thorac Cardiovasc Surg 1983;31:37-40.

2. Kato M, Ohnishi K, Kaneko M, et al. New graftimplanting method for thoracic aortic aneurysm or dissection with a stented graft. Circulation 1996;94:II188-II193.

3. Takahashi K, Maruyama Y, Yoshio T, et al. Rapid expansion of the descending thoracic aortic aneurysm and aneurysm-induced DIC following total arch replacement with a long elephant trunk. Jpn J Cardiovasc Surg 2017;46:130-3.

4. Raffa GM, Pasta S, Gentile G, et al. Early distal remodeling after elephant trunk repair of thoraco-abdominal aortic aneurysms. J Biomech. 2016;49:2398-404. 
5. Higuchi T, Takahashi T, Ishizaka T, et al. Rapid expansion of another downstream aortic aneurysm with the elephant trunk. Gen Thorac Cardiovasc Surg 2010;58:617-9.

6. Fernandez-Bustamante A, Jimeno A. Disseminated

doi: $10.21037 /$ jovs-20-102

Cite this article as: Nakao T, Tsuda S, Kume N, Ikegaya Y, Kitanaka Y. Rapid expansion of the descending aorta following total arch replacement with a FROZENIX-open stent-graft for thoracic aortic aneurysm in a patient with Marfan syndrome: a case report. J Vis Surg 2022;8:25. intravascular coagulopathy in aortic aneurysms. Eur J Intern Med 2005;16:551-60.

7. Kreibich M, Berger T, Rylski B, et al. Aortic reinterventions after the frozen elephant trunk procedure. J Thorac Cardiovasc Surg 2020;159:392-399.e1. 